\title{
Pacientes pediátricos com doença renal crônica em estágio cinco no Brasil: impacto na qualidade de vida
}

\author{
Pediatric patients with stage five chronic kidney disease in Brazil: impact on quality of life \\ Pacientes pediátricos con enfermedad renal crónica en estadio cinco en Brasil: impacto en \\ la calidad de vida
}

Talita Fraga de Azevedo ${ }^{*}$, Ilma Terezinha Palmeira Teixeira ${ }^{1}$, Ana Clara Fraga de Azevedo ${ }^{1}$, Catarina Amaral Cheles ${ }^{1}$, Sabrina Suellem Soares Barbosa ${ }^{1}$, Caio Barbosa Silva ${ }^{1}$, Manuele Trindade Morais ${ }^{1}$, Pedro Câmera de Assis ${ }^{1}$, Mariana Augusta Caixeta Batista Franco ${ }^{1}$, Catharine Silva dos Santos Gomes ${ }^{1}$.

\section{RESUMO}

Objetivo: Analisar o impacto na qualidade de vida dos pacientes pediátricos acometidos por Doença Renal Crônica em estágio cinco no Brasil. Métodos: Realizou-se uma pesquisa bibliográfica do tipo revisão integrativa por meio de coleta de artigos científicos na língua portuguesa e inglesa sem restrição a datas de publicação, através das bases de dados SciELO, Lilacs/lbecs, PubMed e Medline. Foram mantidos todos os aspectos éticos para evitar plágios e cópias. Resultados: Seis estudos foram selecionados para análise e discussão dos dados, de acordo com os critérios de busca e seleção identificados. Apesar das diferenças metodológicas entre os seis artigos, notou-se que em todos eles, a qualidade de vida dos pacientes com Doença Renal Crônica em estágio cinco sofreu impacto negativo. Considerações finais: Conclui-se que a qualidade de vida dos pacientes pediátricos portadores de Doença Renal Crônica em estágio cinco é negativamente impactada, sendo explicado pelas exigências impostas pelo tratamento, e pelas dificuldades e estereótipos relacionados à própria doença. Cada paciente pode atribuir significados diferentes, a partir de uma mesma experiência vivida, sendo imprescindível a escuta individualizada pelo medico e pela equipe de saúde envolvidos.

Palavras-chave: Doença renal crônica, Qualidade de vida, Crianças, Adolescentes.

\begin{abstract}
Objective: To analyze the impact on the quality of life of pediatric patients affected by Stage Five Chronic Kidney Disease in Brazil. Methods: An integrative review was carried out by collecting scientific articles in Portuguese and English without restriction on publication dates, using the SciELO, Lilacs / Ibecs, PubMed and Medline databases. All ethical aspects were maintained to avoid plagiarism and copies. Results: Six studies were selected for analysis and discussion of the data, according to the identified search and selection criteria. Despite the methodological differences between the six articles, it was noted that in all of them, the quality of life of patients with chronic kidney disease in stage five suffered a negative impact. Final considerations: It is concluded that the quality of life of pediatric patients with Chronic Kidney Disease in stage five is negatively impacted, being explained by the demands imposed by the treatment, and by the difficulties and stereotypes related to the disease itself. Each patient can assign different meanings, based on the same lived experience, and individualized listening by the doctor and the health team involved is essential.
\end{abstract}

Keywords: Chronic kidney disease, Quality of life, Children, Adolescent.

\section{RESUMEN}

Objetivo: Analizar el impacto en la calidad de vida de los pacientes pediátricos afectados por la Enfermedad Renal Crónica en Estadio Cinco en Brasil. Métodos: Se realizó una revisión integradora mediante la recopilación de artículos científicos en portugués e inglés sin restricción de fechas de publicación, utilizando las bases de datos SciELO, Lilacs / lbecs, PubMed y Medline. Se mantuvieron todos los aspectos éticos para evitar plagios y copias. Resultados: Se seleccionaron seis estudios para el análisis y discusión de los datos, de acuerdo con los criterios de búsqueda y selección identificados. A pesar de las diferencias metodológicas entre los seis artículos, se observó que en todos ellos la calidad de vida de los pacientes con enfermedad renal crónica en estadio cinco sufrió un impacto negativo. Consideraciones finales: Se concluye que la calidad de vida de los pacientes pediátricos con Enfermedad Renal Crónica en estadio cinco se ve impactada negativamente, lo que se explica por las exigencias que impone el tratamiento, y por las dificultades y estereotipos relacionados con la propia enfermedad. Cada paciente puede asignar diferentes significados, en función de la misma experiencia vivida, y la escucha individualizada por parte del médico y del equipo de salud involucrado es fundamental.

Palabras clave: Enfermedad renal crónica, Calidad de vida, Niños, Adolescentes.

${ }^{1}$ Faculdades Santo Agostinho (FASA), Vitória da Conquista - BA. *E-mail: taah_fraga@hotmail.com 


\section{INTRODUÇÃO}

O Brasil vivencia atualmente um período de transição epidemiológica, onde a morbimortalidade por doenças infecciosas está sendo ultrapassada por doenças crônicas não transmissíveis. Dados da Organização Mundial da Saúde (OMS) apontaram que, no país, no ano de 2017, 73\% das mortes gerais e $17 \%$ das mortes precoces foram devido às doenças crônicas não transmissíveis (CHRISTOFOLETTI M, et al., 2020). As doenças crônicas são definidas como condições médicas ou problemas de saúde com sintomas e incapacidades associadas, que perduram por 3 meses ou mais, exigindo controle e cuidados permanentes devidos a possíveis sequelas que podem ocorrer. Nem sempre são condições incapacitantes, mas podem provocar limitações de atividades (VIEIRA SS, et al., 2009).

Dentre as principais doenças crônicas não transmissíveis, que vêm ganhando força no território brasileiro, estão a hipertensão arterial e a diabetes mellitus, ambos os fatores de risco para a doença renal outra doença crônica não transmissível que vem crescendo bastante no país (MARINHO AWGB, et al., 2017).

A doença renal crônica (DRC) é definida como a presença de anormalidades da estrutura ou função dos rins, presentes por mais de três meses (PORTO JR, et al., 2015), resultante de um grande número de doenças, tanto sistêmicas que danificam os rins quanto intrínsecas a esse órgão (GOLDMAN L e AUSIELLO D, 2018). Apresenta-se como um grave problema de saúde pública, com alta prevalência e incidência, acarretando, além de sofrimento ao paciente, altos custos ao governo. O Censo Brasileiro de Diálise Crônica no Brasil estimou que o país gasta 1,4 bilhão de reais por ano com diálise e transplante (AGUIAR LK, et al., 2020). A prevalência da Doença Renal Crônica na população brasileira ainda é incerta. Estimativas populacionais mais recentes revelam que cerca de 3 a 6 milhões de adultos teriam a doença (MARINHO AWGB, et al., 2017).

A função dos rins é filtrar o sangue, removendo os resíduos tóxicos produzidos no organismo e água e diversas outras substâncias, além disso, produzem hormônios responsáveis pelo controle da pressão arterial, do metabolismo ósseo e da produção de glóbulos vermelhos (SANTOS GLC, et al., 2020).

A Doença Renal Crônica pode ser classificada em cinco estágios, sendo essa classificação, baseada na taxa de filtração glomerular (TFG) e na proteinúria do paciente. O estágio cinco compreende aqueles com TGF $<15 \mathrm{ml} / \mathrm{min} / 1,73 \mathrm{~m}^{2}$, onde os rins perderam sua capacidade total de manter a homeostase corporal, e necessitam, portanto, de terapias substitutivas (PORTO JR, et al., 2015). Essas terapias se subdividem em três tipos: hemodiálise (HD), diálise peritoneal (DP) e transplante renal (GONÇALVES FA, et al., 2015). As metas destes tratamentos são: promover a função renal eficaz, tartar as complicações sistêmicas, manter o equilíbrio hidroeletrolítico dentro dos limites aceitáveis e promover uma melhor qualidade de vida (CLEMENTINO HMC, 2019).

Segundo Tavares APS, et al. (2020), pacientes com Doença Renal Crônica apresentam uma alta carga de sintomas físicos e psicológicos estressante. Os pacientes que são submetidos a procedimentos de diálise precisam ter uma rigidez alimentar e de horário, além de mudanças no contexto familiar, ocupacional e social, que podem interferir em sua qualidade de vida (COSTA KPS, 2012). Essas pessoas estão sujeitas a impactos em suas vidas, bem como na vida de seus familiares, abarcando desde o desconforto físico ao efeito colateral da medicação, passando pelas questões de alteração de hábitos alimentares, infecções, rejeição e diminuição na qualidade de vida (SANTOS GLC, et al., 2020).

A Sociedade Brasileira de Nefrologia (SBN) realizou em 2014, uma pesquisa com 48.834 pacientes, e estimou que aproximadamente 112.000 pacientes diagnosticados com insuficiência renal crônica estavam em programas de diálise ambulatorial. Nessa mesma pesquisa, evidencioou-se também, que o percentual de pacientes com idade inferior ou igual a 18 anos em diálise foi de apenas $1 \%$ do total estudado (FERNANDES JR, et al., 2018). Os estudos sobre a incidência e a prevalência de pacientes diagnosticados com Doença Renal Crônica são feitos, porém poucos existem sobre a população pediátrica em tratamento hemodialítico, especialmente nos quesitos relacionados aos impactos na qualidade, provocados pela patologia e pela hospitalização prolongada (FERNANDES JR, et al., 2018). 
A Doença Renal Crônica acomete, em sua maioria, a população adulta e idosa. Na infância e adolescência, a incidência de acometidos, é menor, porém, ao considerar seus impactos, se deve levar em conta que esses pacientes estão passando por um período de crescimento e desenvolvimento e que cada faixa etária possui suas peculiaridades, tornando o tratamento quase individualizado, mais complexo e mais dispendioso. Dessa forma, as crianças podem sofrer com crises de estresse, depressão, desorganização do dia a dia e baixa autoestima, além de modificarem a maneira como encaram a vida (NOGUEIRA PCK, et al., 2011). Acaba sendo uma experienciência diferente e complexa para elas, já que, após o diagnóstico, precisam conviver com diálise, dietas e drogas medicamentosas (FROTA MA, et al., 2010).

A qualidade de vida das crianças e adolescentes com Doença Renal Crônica é uma questão importante a se levar em consideração. Dessa maneira, o objetivo desse estudo foi entender, através de análises de artigos publicados, o impacto na qualidade de vida dos pacientes pediátricos acometidos por Doença Renal Crônica em estágio cinco no Brasil.

\section{MÉTODOS}

Trata-se de um estudo de revisão integrativa da literatura sobre o impacto na qualidade de vida de pacientes pediátricos portadores de doença renal crônica em estágio cinco no Brasil. Foi criada a seguinte pergunta: "Como essa doença afeta a qualidade de vida desses pacientes?", para alcançar o objetivo proposto.

Foi realizada pesquisa em bancos de dados eletrônicos, sendo eleitos aqueles que continham periódicos que se referissem ao Brasil, dando preferência aos escritos em língua portuguesa, porém, aqueles escritos em inglês também foram analisados. Os bancos de dados pesquisados foram Scientific Electronic Libray Online (SciELO), Literatura Latino-Americana e do Caribe/Índice Bibliográfico Español en Ciencias de la Salud (Lilacs/lbecs), PubMed e Medline, conforme ilustrado no Quadro 1.

A busca foi efetuada no período entre agosto e novembro de 2020. Consideraram-se artigos científicos publicados até agosto de 2020, sem restrição de data anterior a esse período. Empregaram-se as seguintes palavras chaves, em suas variantes na língua inglesa e portuguesa: "Doença Renal Crônica", "Criança" e "Qualidade de Vida".

Quadro 1 - Tabela representativa da metodologia de inclusão dos artigos neste estudo.

\begin{tabular}{|c|c|c|c|}
\hline $\begin{array}{c}\text { Estratégia de busca para as bases de dados, SciELO, Pubmed, Lilacs/IBEC, Medline (Doença } \\
\text { Renal Crônica) AND (Criança) AND (Qualidade de vida) }\end{array}$ \\
\hline $\begin{array}{c}\text { SciELO } \\
9\end{array}$ & $\begin{array}{c}\text { PubMed } \\
0\end{array}$ & $\begin{array}{c}\text { Lilacs } \\
26\end{array}$ & $\begin{array}{c}\text { Medline } \\
261\end{array}$ \\
\hline \multicolumn{5}{|c|}{ Aplicação de critérios de inclusão e exclusão } \\
\hline 7 & 0 & 10 & 6 \\
\hline \multicolumn{5}{|c|}{ Exclusão dos artigos repetidos } \\
\hline 7 & 0 & 8 & 0 \\
\hline 5 & 0 & 1 & 4 \\
\hline
\end{tabular}

Fonte: Azevedo TF, et al., 2021.

Incluíram-se estudos originais realizados em território nacional, publicados em português e inglês, e escritos até agosto de 2020 sem restrição de data anterior a esse período. Os artigos estavam disponíveis em versão eletrônica e tratavam estritamente do impacto da doença renal crônica em estágio cinco nos diversos âmbitos da qualidade de vida dos pacientes pediátricos.

Excluíram-se da análise artigos não originais (editoriais, comentários, revisões, capítulos de livro e cartas) por não contribuírem na discursão do presente estudo. Não foram considerados artigos onde os descritores constavam apenas nas palavras-chave, mas que se distanciavam do objetivo central da pergunta norteadora. Em caso de artigos duplicados - que estavam presentes em mais de uma base de dados-, quatro deles foram excluídos. 
Foram selecionados seis artigos após aplicação dos critérios de inclusão e exclusão, sendo a leitura dos artigos feita pelo título, posteriormente pelo resumo e, por ultimo, pelo texto integral, para verificar quais contemplavam o objetivo de fato. Para análise dos artigos elegidos, foram considerados: ano da publicação e periódico no qual o artigo foi publicado. Posteriormente, cada artigo foi analisado observando-se: objetivos, metodologia, evidências produzidas e aplicabilidade e/ou recomendações.

Por esse trabalho se tratar de uma pesquisa observacional, do tipo revisão integrativa de estudos já publicados na literatura, não foi necessário à submissão ao comitê de ética em pesquisa em seres humanos, já que as informações utilizadas são secundárias e já foram divulgadas, mas seguirá as exigências éticas para evitar o plágio.

\section{RESULTADOS}

Após a análise e seleção dos artigos, foram apresentados no Quadro 2 os seis artigos selecionados de acordo com a cronologia das publicações, seus respectivos autores, seus títulos e os periódicos científicos onde cada texto foi publicado.

Quadro 2 - Artigos selecionados de acordo com ano de publicação e periódico científico.

\begin{tabular}{|c|c|c|}
\hline Autor(es) e ano & Título do artigo & Periódico \\
\hline VIEIRA SS, et al. (2009) & $\begin{array}{c}\text { Doença renal crônica: conhecendo a } \\
\text { experiência da criança }\end{array}$ & $\begin{array}{c}\text { Escola Anna Nery } \\
\text { Revista de Enfermagem }\end{array}$ \\
\hline ABRAHÃO SS, et al. (2010) & $\begin{array}{c}\text { Dificuldades vivenciadas pela família e } \\
\text { pela criança/ adolescente com doença } \\
\text { renal crônica }\end{array}$ & $\begin{array}{c}\text { Jornal Brasileiro de } \\
\text { Nefrologia }\end{array}$ \\
\hline FROTA MA, et al. (2010) & $\begin{array}{c}\text { Qualidade de vida da criança com } \\
\text { Insuficiência Renal Crônica }\end{array}$ & $\begin{array}{c}\text { Escola Anna Nery } \\
\text { Revista de Enfermagem }\end{array}$ \\
\hline TEIXEIRA CG, et al. (2014) & $\begin{array}{c}\text { Impact of chronic kidney disease on } \\
\text { quality of life, lung function, and } \\
\text { functional capacity }\end{array}$ & Jornal de Pediatria \\
\hline ABREU IS, et al. (2015) & $\begin{array}{c}\text { Crianças e adolescentes com } \\
\text { insuficiência renal em hemodiálise: } \\
\text { percepção dos profissionais }\end{array}$ & $\begin{array}{c}\text { Revista Brasileira de } \\
\text { Enfermagem }\end{array}$ \\
\hline ROTELLA AAF, et al. (2020) & $\begin{array}{c}\text { Repercussões emocionais e qualidade } \\
\text { de vida das crianças e adolescentes em } \\
\text { hemodiálise ou após transplante renal }\end{array}$ & $\begin{array}{c}\text { Revista Paulista de } \\
\text { Pediatria }\end{array}$ \\
\hline
\end{tabular}

Fonte: Azevedo TF, et al., 2021.

Ao analisar a metodologia dos seis artigos selecionados, observa-se que, em todos, a forma de coleta se deu através de questionários, diferindo em para quem as perguntas foram direcionadas. Sendo dois deles para apenas os próprios pacientes, dois para os pacientes e cuidadores, um para os profissionais que lidam com esses tipos de pacientes e um somente para os cuidadores.

Observou-se que três artigos procuraram analisar o impacto da Doença Renal Crônica na qualidade de vida das crianças/adolescentes de forma geral, sem focar apenas no prejuízo que um determinado tratamento poderia causar. Um artigo delimitou o impacto em relação à apenas a Diálise Peritonel, um à Hemodiálise e um artigo à Hemodiálise e transplante renal.

Cabe ressaltar que um artigo, além da qualidade de vida, também abordou a função pulmonar e a capacidade funcional dos pacientes com Doença Renal Crônica. Neste caso, consideraram-se, para esse estudo, apenas as questões voltadas à qualidade de vida dessas pessoas. No Quadro 3 são apresentados os resultados dos seis artigos selecionados de acordo com os objetivos, a metodologia, as evidências produzidas e a aplicabilidade e/ou recomendações. 
Quadro 3 - Distribuição dos artigos selecionados, observando-se os seguintes aspectos: objetivos, metodologia, evidências produzidas e aplicabilidade e/ou recomendações.

\begin{tabular}{|c|c|c|c|c|c|}
\hline Autor(es) e ano & Título do artigo & Objetivo(s) & Metodologia & Evidências produzidas & Aplicabilidade e/ou recomendações \\
\hline $\begin{array}{l}\text { VIEIRA SS, et al. } \\
\qquad(2009)\end{array}$ & $\begin{array}{l}\text { Doença renal } \\
\text { crônica: } \\
\text { conhecendo a } \\
\text { experiência da } \\
\text { criança }\end{array}$ & $\begin{array}{l}\text { Compreender a } \\
\text { vivência da criança } \\
\text { com insuficiência renal } \\
\text { crônica e analisar o } \\
\text { significado que ela } \\
\text { atribui a esta vivência }\end{array}$ & $\begin{array}{l}\text { Estudo qualitativo com } \\
8 \text { crianças portadoras } \\
\text { de DRC. Utilizou } \\
\text { entrevista consentida } \\
\text { semi- estruturada }\end{array}$ & $\begin{array}{l}\text { 1) As crianças relatam: medo do } \\
\text { desconhecido; sentem-se mal pela restrição } \\
\text { alimentar e hídrica; sofrem devido o grande } \\
\text { numero de remédios e seus efeitos colaterais; } \\
\text { interrupção das suas atividades diárias } \\
\text { (brincadeiras, sono) e escolares pelo } \\
\text { tratamento ou mal-estar; dor no transcorrer da } \\
\text { doença; sofrem com discriminação e } \\
\text { constantes perguntas sobre sua doença } \\
\text { 2) } 4 \text { crianças almejam o transplante } \\
\text { 3) Essas crianças procuram entender suas } \\
\text { dificuldades e encaram suas restrições como } \\
\text { algo necessário e benéfico, tentam se ver } \\
\text { como uma criança normal }\end{array}$ & $\begin{array}{l}\text { 1) Necessário prosseguir com contínua } \\
\text { transmissão de informações e orientações no } \\
\text { transcorrer do tratamento, investigando suas } \\
\text { dúvidas e novas situações } \\
\text { 2) A maneira como a assistência vem sendo } \\
\text { prestada tem sido questionada por se mostrar } \\
\text { insuficiente para a integralidade do cuidar } \\
\text { 3) Necessário que a equipe de saúde avalie as } \\
\text { atividades cotidianas afetadas e promova } \\
\text { transformações condizentes com a realidade }\end{array}$ \\
\hline $\begin{array}{l}\text { ABRAHÃO SS, } \\
\text { et al. (2010) }\end{array}$ & $\begin{array}{l}\text { Dificuldades } \\
\text { vivenciadas pela } \\
\text { família e pela } \\
\text { criança/ } \\
\text { adolescente com } \\
\text { doença renal } \\
\text { crônica }\end{array}$ & $\begin{array}{l}\text { Analisar as reações e } \\
\text { dificuldades } \\
\text { vivenciadas pela } \\
\text { família e pela criança/ } \\
\text { adolescente com DRC } \\
\text { para a realização da } \\
\text { Diálise Peritoneal }\end{array}$ & $\begin{array}{l}\text { Estudo descritivo com } \\
\text { os cuidadores de } 30 \\
\text { crianças e } \\
\text { adolescentes com } \\
\text { DRC. Foi utilizado um } \\
\text { roteiro de visita } \\
\text { domiciliar e um } \\
\text { questionário }\end{array}$ & $\begin{array}{l}\text { 1) Os cuidadores relataram que as } \\
\text { crianças/adolescentes se queixam de ter que } \\
\text { fazer a diálise pelo uso de máscara, dor e } \\
\text { prurido no orifício; de deixar de fazer outras } \\
\text { atividades (brincar, viajar, ir à escola, dormir } \\
\text { fora) por conta do procedimento; do grande } \\
\text { número de medicamentos e seus efeitos } \\
\text { colaterais; da restrição dietética } \\
\text { 2) } 2 \text { cuidadores relataram situações de } \\
\text { depressão e tristeza; } 1 \text { relatou vergonha do } \\
\text { cateter na barriga; } 3 \text { relataram ansiedade e } \\
\text { nervosismo; } 2 \text { relataram inquietude }\end{array}$ & $\begin{array}{l}\text { 1) Criar estratégias para reduzir a má adesão } \\
\text { medicamentosa como, por exemplo, seu uso } \\
\text { próximo às refeições ou junto a algum alimento do } \\
\text { gosto do paciente } \\
\text { 2) É possível uma adaptação à situação com } \\
\text { preservação de uma satisfatória qualidade de vida } \\
\text { segundo a percepção do paciente e/ou da família }\end{array}$ \\
\hline $\begin{array}{l}\text { FROTA MA, et } \\
\text { al. (2010) }\end{array}$ & $\begin{array}{c}\text { Qualidade de } \\
\text { vida da criança } \\
\text { com } \\
\text { Insuficiência } \\
\text { Renal Crônica }\end{array}$ & $\begin{array}{l}\text { Avaliar a qualidade de } \\
\text { vida de crianças com } \\
\text { insuficiência renal } \\
\text { crônica e identificar os } \\
\text { domínios mais } \\
\text { relevantes }\end{array}$ & $\begin{array}{c}\text { Estudo descritivo, de } \\
\text { abordagem qualitativa } \\
\text { e quantitativa, com } 13 \\
\text { crianças com } \\
\text { diagnóstico de IRC. } \\
\text { Tecnica: aplicação do } \\
\text { Autoquestionnaire } \\
\text { Qualité de Vie Enfant } \\
\text { Imagé (AUQEI) }\end{array}$ & $\begin{array}{l}\text { 1) } 8 \text { crianças mencionaram que o processo } \\
\text { da diálise é doloroso. } \\
\text { 2) Algumas reclamaram da hospitalização, } \\
\text { por causar ansiedade e restringir o apoio } \\
\text { familiar } \\
\text { 3) O ato de brincar e ir à escola são } \\
\text { comprometidos pelos limites do tratamento } \\
\text { 4) } 1 \text { criança relatou sofrer discriminação } \\
\text { 5) O transplante foi mencionado como algo } \\
\text { que traria felicidade e uma melhor qualidade } \\
\text { de vida. } \\
\text { 6) Relataram a importância do apoio familiar }\end{array}$ & $\begin{array}{l}\text { 1) Necessidade do desenvolvimento de ações em } \\
\text { que a criança possa se sentir mais independente, } \\
\text { capaz de realizar atividades do cotidiano, } \\
\text { relacionadas, principalmente, ao lazer } \\
\text { 2) A criança com IRC, em sua percepção de } \\
\text { mundo, é feliz, apesar de suas limitações e das } \\
\text { inúmeras complicações a que está sujeita }\end{array}$ \\
\hline
\end{tabular}




\begin{tabular}{|c|c|c|c|c|c|}
\hline Autor(es) e ano & Título do artigo & Objetivo(s) & Metodologia & Evidências produzidas & Aplicabilidade e/ou recomendações \\
\hline $\begin{array}{l}\text { TEIXEIRA CG, et } \\
\text { al. (2014) }\end{array}$ & $\begin{array}{l}\text { Impact of chronic } \\
\text { kidney disease } \\
\text { on quality of life, } \\
\text { lung function, } \\
\text { and functional } \\
\text { capacity }\end{array}$ & $\begin{array}{l}\text { Avaliar repercussões } \\
\text { da DCR sobre a } \\
\text { qualidade de vida na } \\
\text { percepção das } \\
\text { crianças e dos pais, } \\
\text { força muscular } \\
\text { respiratória, função } \\
\text { pulmonar e } \\
\text { capacidade funcional } \\
\text { em crianças e } \\
\text { adolescentes }\end{array}$ & $\begin{array}{c}\text { Estudo observacional } \\
\text { do tipo corte } \\
\text { transversal com } 40 \\
\text { crianças e } \\
\text { adolescentes com } \\
\text { DRC. Foi aplicado um } \\
\text { questionário de } \\
\text { qualidade de vida } \\
\text { (PedsQL) para } \\
\text { pacientes e um de } \\
\text { seus pais }\end{array}$ & $\begin{array}{l}\text { 1) Ao comparar a qualidade de vida e o tipo } \\
\text { de tratamento evidenciou-se que os } \\
\text { transplantados obtiveram um maior escore, } \\
\text { quando comparados ao grupo que realizava } \\
\text { hemodiálise } \\
\text { 2) Aquelas que realizavam atividade física } \\
\text { regular apresentavam uma melhor qualidade } \\
\text { de vida, quando comparadas às sedentárias }\end{array}$ & $\begin{array}{l}\text { Observou-se redução significativa na qualidade de } \\
\text { vida em crianças com DRC influenciadas pelo tipo } \\
\text { de tratamento e sedentarismo. }\end{array}$ \\
\hline $\begin{array}{l}\text { ABREU IS, et al. } \\
\qquad(2015)\end{array}$ & $\begin{array}{l}\text { Crianças e } \\
\text { adolescentes } \\
\text { com } \\
\text { insuficiência } \\
\text { renal em } \\
\text { hemodiálise: } \\
\text { percepção dos } \\
\text { profissionais }\end{array}$ & $\begin{array}{l}\text { Identificar aspectos } \\
\text { impactantes na } \\
\text { qualidade de vida de } \\
\text { crianças e } \\
\text { adolescentes com IRC } \\
\text { crônica em } \\
\text { hemodiálise }\end{array}$ & $\begin{array}{l}\text { Pesquisa qualitativa } \\
\text { realizada com } 12 \\
\text { profissionais da } \\
\text { saúde. Aplicação de } \\
\text { um questionário sobre } \\
\text { qualidade de vida de } \\
\text { crianças e } \\
\text { adolescentes com IRC } \\
\text { em hemodiálise }\end{array}$ & $\begin{array}{l}\text { 1) O diagnóstico da doença causa um grande } \\
\text { impacto, afetando seu cotidiano e gerando } \\
\text { medo, insegurança, raiva e negação } \\
\text { 2) O suporte familiar é importante, porém } \\
\text { esse apoio nem sempre é percebido, } \\
\text { principalmente aos adolescentes } \\
\text { 3) Negligência de aspectos importantes } \\
\text { referentes a outras esferas de suas vidas } \\
\text { devido à centralização de suas atividades em } \\
\text { torno do tratamento } \\
\text { 4) Expectativa dos pacientes em relação ao } \\
\text { transplante renal e sua frustação quando não } \\
\text { se concretiza } \\
\text { 5) Impacto na socialização. }\end{array}$ & $\begin{array}{l}\text { 1) Incentivo dos profissionais da unidade de diálise } \\
\text { para os pais sobre a importância da continuidade } \\
\text { das atividades escolares de seus filhos } \\
\text { 2) O transplante renal é considerado o melhor } \\
\text { tratamento para a IRC comparado a outros } \\
\text { métodos dialíticos. A sobrevida de pacientes } \\
\text { transplantados, adultos ou pediátricos é superior à } \\
\text { daqueles em diálise, o que de fato lhes confere } \\
\text { melhor qualidade de vida }\end{array}$ \\
\hline $\begin{array}{l}\text { ROTELLA AAF, } \\
\text { et al. (2020) }\end{array}$ & $\begin{array}{l}\text { Repercussões } \\
\text { emocionais e } \\
\text { qualidade de } \\
\text { vida das } \\
\text { crianças e } \\
\text { adolescentes em } \\
\text { hemodiálise ou } \\
\text { após transplante } \\
\text { renal }\end{array}$ & $\begin{array}{c}\text { Investigar as } \\
\text { repercussões } \\
\text { emocionais e a } \\
\text { qualidade de vida (QV) } \\
\text { associadas à doença } \\
\text { renal crônica em } \\
\text { crianças e } \\
\text { adolescentes } \\
\text { submetidos à } \\
\text { hemodiálise ou ao } \\
\text { transplante } \\
\text { renal }\end{array}$ & $\begin{array}{l}\text { Pesquisa qualitativa } \\
\text { realizada com } 96 \\
\text { indivíduos, metade } \\
\text { deles crianças e } \\
\text { adolescentes e, a } \\
\text { outra, seus } \\
\text { respectivos } \\
\text { cuidadores }\end{array}$ & $\begin{array}{l}\text { 1) A qualidade de vida dos pacientes } \\
\text { transplantados é melhor do que naqueles que } \\
\text { estão em diálise, principalmente no quesito } \\
\text { capacidade física } \\
\text { 2) Os quesitos emocional e social } \\
\text { permanecem comprometidos de forma igual } \\
\text { 3) Cuidadores referiram comprometimento } \\
\text { escolar }\end{array}$ & $\begin{array}{l}\text { 1) As pessoas podem interagir de maneiras } \\
\text { diferentes ao serem acometidas pela DRC ou } \\
\text { quando se submetem ao transplante renal } \\
\text { 2) O transplante renal pode auxiliar o paciente } \\
\text { do ponto de vista clínico e psíquico, mas não } \\
\text { obrigatoriamente, e não se deve associá-lo à } \\
\text { solução }\end{array}$ \\
\hline
\end{tabular}

Fonte: Azevedo TF, et al., 2021. 


\section{DISCUSSÃO}

Pode-se verificar o quão escassos são os estudos abordando o impacto que a Doença Renal Crônica (DRC) em estágio cinco tem na qualidade de vida de crianças e adolescentes. Mesmo não exigindo data mínima para a seleção dos artigos, conseguiram-se poucos materiais para serem analisados. Fato esse controverso ao relacionar com a importância do tema e com a grande necessidade de estabelecer caminhos para melhorar a qualidade de vida desses pacientes.

Segundo Gonçalves FA, et al. (2015) o termo qualidade de vida, quando se leva em conta a saúde, abrange aspectos físicos, sociais e emocionais causadas por uma doença ou tratamento. Apesar de algumas diferenças metodológicas entre os seis artigos, notou-se que em todos eles, a qualidade de vida dos pacientes com Doença Renal Crônica em estágio cinco sofreu impacto negativo significativo.

A cronicidade e o risco de complicações da Doença Renal Crônica levam ao controle e cuidados constantes para que se avitem sequelas, fazendo com que as crianças sofram privações e limitações pelas exigências de seus tratamentos, impactanto em suas atividades rotineiras devido às mudanças no estilo e qualidade de vida. Esses fatores podem acabar gerando uma instabilidade emocional em toda a família, bem como comprometimentos no desenvolvimento físico, emocional e psicológico da criança (SIMÕES $\mathrm{KCR}$, et al., 2020). Cada paciente vive e se relaciona com a doença de maneira única e pessoal, dependendo de vários fatores, como o psicológico, as condições ambientais, sociais e familiares, além da resposta da equipe de saúde (CLEMENTINO HMC, 2019).

A desfavorável repercussão no ato de brincar, explicado pelas exigências impostas pelo tratamento e pelas dificuldades inerentes à própria doença, foi o mais enfatizado, estando presente nos seis artigos analisados. Por se tratar de pacientes pediátricos, a predominância desse resultado seria esperada. Carvalho MC (2016) afirma que o brincar é fundamental para o desenvolvimento humano nesse período, colaborando para uma boa saúde mental e física, e facilitando o processo de socialização, comunicação, expressão e construção do conhecimento.

Neste sentido, observa-se que junto com esse impacto, problemas secundários podem surgir. A criança pode, por exemplo, tornar-se sedentária, depressiva e/ou nervosa. Todos esses aspectos afetam não apenas a qualidade de vida como também o próprio processo de tratamento. Neste contexto, a criança com Doença Renal Crônica precisa ser submetida à participação em atividades lúdicas, com o intuito de viver sua infância e se distanciar de certa forma do processo da doença. Baixos níveis de atividade física também foram relacionados com uma pior qualidade de vida e um pior desempenho físico (TEIXEIRA CG, et al., 2014).

Além das brincadeiras, a dieta específica foi apontada por dois artigos como impactante na qualidade de vida. Observou-se que a necessidade de uma restrição alimentar e hídrica para o bom controle da doença, não deixa as crianças/adolescentes satisfeitos. De acordo com Abrahão SS, et al. (2010), é um sacrifício para esses pacientes não poder comer chocolate, salgadinhos, biscoitos recheados e tomar um refrigerante. Não poder ingerir ou ver seus amigos comendo, podem causar frustração e sentimentos negativos. É muito mais difícil para a criança não comer, não brincar ou realizar outras coisas quando ela está em um meio em que esses fatos estão acontecendo (VIEIRA SS, et al., 2009).

Como toda doença crônica, é natural que o paciente e a familia ocupem seu tempo em torno do tratamento, negligenciando outras áreas do dia a dia (ROTELLA AAF, et al, 2019). O impacto escolar emergiu em três artigos como tema digno de atenção. Isso pode ocorrer devido à frequência das sessões de hemodiálise e o período que os participantes ficam no hospital (SOUZA TT, et al., 2019), além da discriminação que as crianças sofrem, seja pelos cateteres, pela pele pálida ou pela baixa estatura, característicos da doença (FROTA MA, et al., 2010).

Alguns pacientes sofrem para revelar que são renais crônicos, sentem vergonha das fístulas arteriovenosas no braço, podem apresentar atraso no desenvolvimento de caracteres sexuais, deformidades ósseas e cicatrizes, que despertam a curiosidade alheia, e pode gerar situações constrangedoras e agravar o isolamento (ROTTELA AAF, et al., 2019). Apesar disso, desses três artigos, 
um citou a grande importância escolar nessa faixa etária, já que as relações sociais acontecem nesse espaço, e reforçou o papel dos profissionais em orientar e promover a continuidade educacional (ABREU IS, et al., 2015).

O contexto hospitalar e a rotina de diversos procedimentos invasivos, pode fazer a criança sentir-se inserida em um ambiente desconhecido, gerando sentimentos negativos em relação às mudanças ocorridas (SOUZA TT, et al., 2019). O medo do desconhecido, a grande quantidade de remédios e seus efeitos colaterais, e a dor dos procedimentos também foram apontados no impacto da qualidade de vida dos portadores de Doença Renal Crônica em estágio cinco. As crianças, por terem mais dificuldade em aceitarem os medicamentos e compreenderem a sua importância, acaba rejeitando, jogando fora ou vomitando. Tendo isso em vista, é importante que se crie mecanismos para reduzir a má adesão medicamentosa, como por exemplo seu uso próximo às refeições ou junto a algum alimento do gosto do paciente (ABRAHÃO SS, et al., 2010).

Apesar disso, algumas destas crianças possuem uma percepção mais madura sobre a vida e a doença em relação a crianças normais, procurando enxergar essas dificuldades como necessárias para uma boa qualidade de vida (VIEIRA SS, et al., 2009). Esses pacientes preocupam-se, ainda, com os seus pais e irmãos, por terem abdicado de muitos de seus sonhos para cuidar deles, em vê-los sobrecarregados e em diminuir as atividades de lazer da família (VIEIRA SS, et al., 2009).

É essencial reforçar a importância do apoio familiar nesse momento, pois esse suporte é fundamental e relevante para a recuperação e manutenção da saúde das crianças e adolescentes, permitindo que se sintam mais protegidos em um momento onde estão frágeis e sensíveis (ABREU IS, et al., 2015). Dois estudos mostraram o quanto as crianças ficam felizes quando seus pais estão ao seu lado e o quanto elas reclamam quando, por algum procedimento, precisam ficar longe deles. Os pacientes compartilham com seus parentes os medos e buscam apoio e ajuda, pois essa situação requer adaptação individual e familiar (FROTA MA, et al., 2010).

Apesar dessa importância, Abreu IS, et al. (2015), ressalta em seu estudo que esse apoio não foi percebido, principalmente no caso de alguns adolescentes, mostrando o quanto as relações afetivas familiares podem ser frágeis, gerando tristeza, medo, estresse e insegurança para esses pacientes.

É evidente que adolescentes com Doença Renal Crônica vivem embates considerados normais para sua faixa etária, como, por exemplo, conflitos em busca da autonomia que culminam com a redefinição do indivíduo para o seu ingresso na vida adulta. Se não bastasse, esses conflitos são agravados pela dificuldade de manejo da doença e pelos sentimentos de revolta e repúdio à condição clínica e seu tratamento. Nesses pacientes, ocorre frequentemente uma piora do controle clínico e da adesão à terapêutica. Os efeitos colaterais das medicações, as múltiplas ausências escolares e as alterações psicossociais, notadamente baixa autoestima, resultam em retardo de crescimento e desenvolvimento, atraso puberal e pior desempenho cognitive (MARCIANO RC, et al., 2010).

Por fim, todos os seis artigos convergiram seus resultados em relação à grande expectativa que esses pacientes possuem em conseguirem um transplante renal. Nos pacientes transplantados, alguns pontos da Qualidade de Vida se equipararam aos daqueles submetidos à hemodiálise, mas percebe-se que há algumas modificações vantajosas além da maior sobrevida em relação à diálise (ROTELLA AAF, et al., 2019). Eles veem, neste tratamento, a possibilidade de uma vida normal, vivendo suas vidas sem as restrições impostas pela doença. Porém, atrelado a isso, está a grande frustração dessas crianças/adolescentes quando não conseguem, e por isso, esse assunto precisa ser abordado com eles de maneira bastante cautelosa e realista a fim de evitar criar expectativas insustentáveis (ABREU IS, et al., 2015).

É impressindível o encaminhamento precoce dos pacientes para unidades de serviço especializado, composto por uma equipe multidiciplinar, a fim de haja um preparo e suporte pisicológico tanto do doente quanto da sua família evitando um início de tratamento traumático e escolhendo a melhor modalidade de Terapia Renal Substitutiva (TRS): Transplante Renal, Hemodiálise e Diálise Peritoneal (FERNANDES JR, et al., 2018). 
Uma maior aproximação do médico responsável pelo paciente e de toda a equipe de saúde envolvida é necessária para que se possa ouvir e compreender as limitações e os impactos que a doença gera na vida deles e, assim, possibilitar a ajuda na resolução dos problemas. Deve-se lembrar de que cada paciente pode atribuir significados diferentes a partir de uma mesma experiência vivida, sendo imprescindível a escuta individualizada.

\section{CONSIDERAÇÕES FINAIS}

Seja através de restrições dietéticas e hídricas, do impacto no lazer e na educação, da discriminação sofrida, da dor dos procedimentos terapêuticos, dos efeitos colaterais dos medicamentos, ou da frustração por não conseguir um transplante renal, conclui-se, após este estudo, que, de fato, a qualidade de vida dos pacientes pediátricos portadores de Doença Renal Crônica em estágio cinco é negativamente impactada. É importante salientar que foram encontrados para a análise poucos artigos que contemplavam o objetivo proposto deste estudo, apesar da grande importância do tema. Neste sentido, percebe-se que o Brasil precisa avançar na discursão dessa questão, pois é só conhecendo a realidade que se pode pensar e criar estratégias que garantam a esses pacientes uma melhor qualidade de vida.

\section{REFERÊNCIAS}

1. ABRAHÃO SS, et al. Dificuldades vivenciadas pela família e pela criança/adolescente com doença renal crônica. Brazilian Journal of Nephrology, 2010; 32(1): 18-22.

2. ABREU IS, et al. Crianças e adolescentes com insuficiência renal em hemodiálise: percepção dos profissionais. Revista Brasileira de Enfermagem, 2015; 68(6): 1020-1026.

3. AGUIAR LK, et al. Fatores associados à doença renal crônica: inquérico epidemiológico da Pesquisa Nacional de Saúde. Revista brasileira de epidemiologia, 2020; 23: 15p.

4. CARVALHO MC. A importância do brincar na construção de conhecimentos de crianças na pré-escola. Dissertação (Mestrado em Docência e Gestão da Educação) - Universidade Fernando Pessoa, Porto, 2016; 145p.

5. CHRISTOFOLETTI M, et al. Simultaneidade de doenças crônicas não transmissíveis em 2013 nas capitais brasileiras: prevalência e perfil sociodemográfico. Epidemiologia e Serviços de Saúde, 2020; 29: e201848.

6. CLEMENTINO, HMC. O transplante renal como uma proposta de melhoria na vida de crianças e adolescentes em diálise crônica, no Maranhão. Artigo científico (Curso de Medicina) - Universidade Federal do Maranhão, Pinheiro, 2019; 51p.

7. COSTA KPS. Adesão de pacientes portadores de insuficiência renal crônica à terapia dialítica. Monografia (Curso de Pós-graduação em Nefrologia) - Universidade Paulista, Recife, 2012; 32p.

8. FERNANDES JR, et al. Depressão e qualidade de vida em crianças e adolescentes diagnosticados com doença renal crônica em hemodiálise. Revista Sociedade Brasileira de Psicologia Hospitalar, 2018; 21 (2): 44-60.

9. FROTA MA, et al. Qualidade de vida da criança com insuficiência renal crônica. Escola Anna Nery, 2010; 14(3): 527533.

10. GOLDMAN L, AUSIELLO D. Goldman-Cecil Medicina Interna. 25a ed. Rio de Janeiro: Elsevier, 2018; 3112p.

11. GONÇALVES FA, et al. Qualidade de vida de pacientes renais crônicos em hemodiálise ou diálise peritoneal: estudo comparativo em um serviço de referência de Curitiba-PR. Brazilian Journal of Nephrology, 2015; 37(4): 467-474.

12. GUYTON AC, HALL JE. Guyton \& Hall Tratado de Fisiologia Médica. 13a ed. Rio de Janeiro: GEN Guanabara Koogan, 2017; 1176p.

13. KLIEGMAN RM, et al. Nelson Tratado de pediatría. 21a ed. Espanha: Elsevier, 2020; 4336p.

14. KOEPPEN BM, STANTON BA. Berne \& Levy Fisiologia. 7a ed. Rio de Janeiro: Elsevier, 2018; 880p.

15. MARCIANO RC, et al. Transtornos mentais e qualidade de vida em crianças e adolescentes com doença renal crônica e em seus cuidadores. Brazilian Journal of Nephrology, 2010; 32(3): 316-322.

16. MARINHO AWGB, et al. Prevalência de doença renal crônica em adultos no Brasil: revisão sistemática da literatura. Cadernos Saúde Coletiva, 2017 25(3): 379-388.

17. NOGUEIRA PCK, et al. Prevalência estimada da doença renal crônica terminal em crianças no Estado de São Paulo. Revista da Associação Médica Brasileira, 2011; 57(4): 443-449.

18. PORTO JR, et al. Avaliação da função renal na doença renal crônica. Revista Brasileira de Análises Clínicas, 2017; 49(1): 26-35.

19. ROTELLA AAF, et al. Repercussões emocionais e qualidade de vida das crianças e adolescentes em hemodiálise ou após transplante renal. Revista Paulista de Pediatria, 2020; 38: e2018221.

20. SIMOES KCR, et al. Vozes à infância silenciada: impactos da hospitalização e hemodiálise à escolarização de crianças com doença renal crônica. Revista Educação Especial, 2020; 33: 52-77.

21. SANTOS GLC, et al. A percepção da pessoa sobre sua condição enquanto doente renal crônico em hemodiálise. Rev. Pesqui.(Univ. Fed. Estado Rio J., Online), 2020; 12: 636-641.

22. TAVARES APS, et al. Cuidados de suporte renal: uma atualização da situação atual dos cuidados paliativos em pacientes com DRC. Brazilian Journal of Nephrology,2020; 43(1): 74-87.

23. TEIXEIRA CG, et al. Impact of chronic kidney disease on quality of life, lung function, and functional capacity. Jornal de pediatria, 2014; 90(6): 580-586.

24. VIEIRA, SS, et al. Doença renal crônica: conhecendo a experiência da criança. Escola Anna Nery, 2009; 13(1): 7483. 